ИЗВЕСТИЯ АКАДЕМИИ НАУК ЭСТОНСКОП ССР. ТОМ 30 ФИЗИКА * МАТЕМАТИКА. 1981, № 3

\title{
О РАЗРЕШИМОСТИ ЗАДАЧИ НЕЛИНЕЙНОГО ПРОГРАММИРОВАНИЯ СО СЛУЧАЙНМ ПАРАМЕТРОМ
}

\author{
(Представил Н. Алумяэ)
}

Рассматривается задача нелинейного программирования, зависящая от случайного вектор-параметра. Оцениваются вероятность разрешимости этой задачи и вероятность, что точка решения находится от определенной точки на меньшем расстоянии, чем заданное положительное число. Эти вопросы изучались автором ранее для экстремальных задач без ограничений и с ограничениями типа равенств в работе [ $\left.{ }^{1}\right]$. На результаты [1] существенно опирается и настоящая статья.

Пусть требуется оценить вероятность разрешимости задачи

$$
\min _{x}\left\{f(x, \xi) \mid g^{1} \ldots m(x, \xi) \leqslant 0\right\},
$$

где $f: R^{n} \times R^{s} \rightarrow R^{1}, g^{1 \ldots m}=\left(g_{1} \ldots g_{m}\right)^{\mathrm{T}}: R^{n} \times R^{s} \rightarrow R^{m}, \quad$ параметр $\xi \in R^{s}$ случайный, $f(x, \xi)$ и $g^{4 \ldots m}(x, \xi)$ измеримы по $\xi$ при любом $x \in R^{n}$, и получить некоторую информацию о распределении ее точки решения. Под точкой решения (или решением) везде далее будем подразумевать точку локального минимума. Наряду с задачей (1) рассмотрим задачу

$$
\min \left\{E f(x, \xi) \mid E g^{1} \ldots m(x, \xi) \leqslant 0\right\},
$$

где $E$ обозначает математическое ожидание по $\xi$ при фиксированном $x$. Допустим, что задача (2) имеет решение $x^{*}$, т. е. $\left.\operatorname{Eg}^{1 \ldots}, x^{*}, \xi\right) \leqslant 0$ и для некоторого $\delta>0$ выполняется $E f\left(x^{*}, \xi\right) \leqslant E f(x, \xi)$, если $\left\|x-x^{*}\right\| \leqslant \delta$ и $E g^{1 \ldots m}(x, \xi) \leqslant 0$. Не ограничивая общности, будем считать, что $E g_{i}\left(x^{*}, \xi\right)=0$ при $i=1, \ldots, m_{1}$ и $E g_{i}\left(x^{*}, \xi\right)<0$ при $i=m_{1}+1, \ldots$ $\ldots, m$. В таком случае $x^{*}$ является также решением задачи

$$
\min \left\{E f(x, \xi) \mid E g^{1} \ldots m_{1}(x, \xi)=0\right\} .
$$

Введем следующие предположения:

1. Функция $E g^{1 \ldots m}(x, \xi)$ дифференцируема, функции $E f(x, \xi)$ и $E g^{1_{r} . . m_{1}}(x, \xi)$ дважды дифференцируемы в точке $x^{*}$ и ранг матрицы $\left(E f^{\prime}{ }_{x}\left(x^{*}, \xi\right) E g_{1 x^{\prime}}\left(x^{*}, \xi\right) \ldots E g_{m_{1}} x^{\prime}\left(x^{*}, \xi\right)\right)$ равен $m_{1}$.

Это предположение достаточно для существования вектора $\lambda^{*}=$ $=\left(\lambda_{1}{ }^{*} \ldots \lambda_{m}{ }^{*}\right)^{\mathrm{T}}$ такого, что $L^{\prime}{ }_{x}\left(x^{*}, \lambda^{*}\right)=0$, где

$L(x, \lambda)=E f(x, \xi)+\sum_{i=1}^{m_{1}} \lambda_{i} E g_{i}(x, \xi), \quad$ и постоянной $\mathrm{Q}>0$ такой, что $\left\|\left[E g^{1 \ldots m_{x}^{\prime}}\left(x^{*}, \xi\right)\right]^{\mathrm{T}} v\right\| \geqslant \varrho\|v\| \quad$ для каждого $v \in R^{m_{\mathrm{t}}},\left[{ }^{2}\right]$. 
2. Матрица $L_{x x}^{\prime \prime}\left(x^{*}, \lambda^{*}\right)=E f_{x x}^{\prime \prime}\left(x^{*}, \xi\right)+\sum_{i=1}^{m_{1}} \lambda_{i}^{*} E g_{i x x}^{\prime \prime}\left(x^{*}, \xi\right)$ положительно определена, т. е. для некоторого $M>0$ и для всех $u \in R^{n}$ выполняется $u^{\mathrm{T}} L_{x x^{\prime \prime}}\left(x^{*}, \lambda^{*}\right) u \geqslant M\|u\|^{2}$.

3. Функции $f(x, \xi)$ и $g^{1 \ldots m_{1}}(x, \xi)$ дважды дифференцируемы по $x$ для почти всех $\xi$, причем

$$
\begin{gathered}
\left\|f_{x x}^{\prime \prime}\left(x^{1}, \xi\right)-f_{x x}^{\prime \prime}\left(x^{2}, \xi\right)\right\| \leqslant C_{1}(\xi)\left\|x^{1}-x^{2}\right\|, \\
\| g^{1 \ldots m_{1} \prime \prime}\left(x^{1}, \xi\right)-g_{x x}^{1 \ldots m_{1} \prime \prime}\left(\left(x^{2}, \xi\right)\left\|\leqslant C_{2}(\xi)\right\| x^{1}-x^{2} \|,\right. \\
\left\|g^{1 \ldots m_{1} \prime \prime}(x, \xi)\right\| \leqslant R(\xi)
\end{gathered}
$$

при любых $x, x^{1}, x^{2} \in R^{n}$.

4. $E C_{1}(\xi), E C_{2}(\xi), E R(\xi)$ и дисперсии $\sigma^{2}\left[C_{1}(\xi)\right], \sigma^{2}\left[C_{2}(\xi)\right], \sigma^{2}[R(\xi)]$ конечны.

5. $E\left\|g^{1 \ldots m_{1}^{\prime}} x_{x}^{\prime}\left(x^{*}, \xi\right)-E g_{x}^{1 \ldots m_{1}^{\prime}}\left(x^{*}, \xi\right)\right\|^{2}, \quad E\left\|g_{x x}^{1 \ldots m_{1} \prime \prime}\left(x^{*}, \xi\right)-E g_{x x}^{1 \ldots m_{1} \prime \prime}\left(x^{*}, \xi\right)\right\|^{2}$, $E\left\|f_{x x}^{\prime \prime}\left(x^{*}, \xi\right)-E f_{x x}^{\prime \prime}\left(x^{*}, \xi\right)\right\|^{2}, \quad \sigma^{2}\left[f_{x_{t}}^{\prime}\left(x^{*}, \xi\right)\right], \quad \sigma^{2}\left[g_{j}\left(x^{*}, \xi\right)\right], \quad i=1, \ldots, n$, $j=1, \ldots, m$, конечны.

6. Для почти всех $\xi$ имеет место $\left\|g^{m_{1}+1 \ldots m}\left(x^{1}, \xi\right)-g^{m_{1}+1 \ldots m}\left(x^{2}, \xi\right)\right\| \leqslant$ $\leqslant K\left\|x^{1}-x^{2}\right\|$ при $x^{1}, x^{2},\left\|x^{1}-x^{*}\right\| \leqslant \delta, \quad\left\|x^{2}-x^{*}\right\| \leqslant \delta$, и $g^{m_{1}+1 \ldots m}(x, \xi)$ дифференцируема по $x$.

Введем следующие обозначения:

$$
\begin{gathered}
D_{1}=\left[\sqrt{E\left\|f_{x x}^{\prime \prime}\left(x^{*}, \xi\right)-E f_{x x}^{\prime \prime}\left(x^{*}, \xi\right)\right\|^{2}}+\right. \\
\left.+\left\|\lambda^{*}\right\| \sqrt{E\left\|g_{x x}^{1 \ldots m_{1} \prime \prime}\left(x^{*}, \xi\right)-E g^{1 \ldots m_{1}^{\prime \prime \prime}}\left(x^{*}, \xi\right)\right\|^{2}}\right]^{2}, \\
D_{2}=E\left\|g_{x}^{1 \ldots m_{1}^{\prime}}\left(x^{*}, \xi\right)-E g_{x}^{1 \ldots m_{1}^{\prime}}\left(x^{*}, \xi\right)\right\|^{2}, \\
D_{3}=\sum_{i=1}^{n} \sigma^{2}\left[f_{x_{t}}^{\prime}\left(x^{*}, \xi\right)\right], \quad D_{4}=\sum_{j=1}^{m_{1}} \sigma^{2}\left[g_{j}\left(x^{*}, \xi\right)\right], \\
\psi_{1}=\sigma^{2}\left[C_{1}(\xi)\right], \quad \psi_{2}=\sigma^{2}\left[C_{2}(\xi)\right], \quad \psi_{3}=\sigma^{2}[R(\xi)] .
\end{gathered}
$$

Т е о рем а. Пусть найдутся постоянные $\gamma_{1}, \gamma_{2}, \ldots, \gamma_{6}, 0^{\circ}<\gamma_{1}<M$, $0<\gamma_{2}<\varrho, \gamma_{3}>0, \gamma_{4}>0, \gamma_{5}>0,0^{\circ}<\gamma_{6}<1$ такие, что выражение

$$
\begin{gathered}
q\left(\gamma_{1}, \ldots, \gamma_{6}\right)=1-\left[\frac{D_{1}}{\gamma_{1}^{2}}+\frac{D_{2}}{\gamma_{2}^{2}}+\frac{\psi_{1}}{\gamma_{3}^{2}}+\frac{\psi_{2}}{\gamma_{4}^{2}}+\frac{\psi_{3}}{\gamma_{5}^{2}}+\right. \\
\left.+\frac{\sum_{m_{1}+1}^{m} \sigma^{2}\left[g_{i}\left(x^{*}, \xi\right)\right]}{\left(1-\gamma_{6}\right)^{2}\left|\max _{i=m_{1}+1, \ldots, m} E g_{i}\left(x^{*}, \xi\right)\right|^{2}}\right]- \\
c_{1}\left(\sqrt[3]{D_{3}}+\sqrt[3]{\left\|\lambda^{*}\right\|^{2} D_{2}}+\sqrt[3]{D_{4}}\right)^{3}
\end{gathered}
$$
где

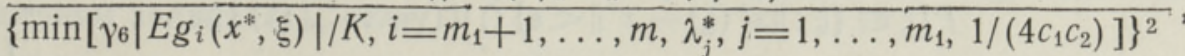

$$
\begin{aligned}
& c_{1}=\max \left[\alpha_{1}+\alpha_{1}^{2} \alpha_{2}^{2} \alpha_{3}, \alpha_{3}\right]+\alpha_{1} \alpha_{2} \alpha_{3}, \\
& \alpha_{1}=1 /\left(M-\gamma_{1}\right), \quad \alpha_{2}=\left\|E g^{1 \ldots m_{1}^{\prime}}\left(x^{*}, \xi\right)\right\|+\gamma_{2},
\end{aligned}
$$




$$
\begin{aligned}
& \alpha_{3}=\left(\left\|L_{x x}^{\prime \prime}\left(x^{*}, \lambda^{*}\right)\right\|+\gamma_{1}\right) /\left(\varrho-\gamma_{2}\right)^{2}, \\
& c_{2}=E C_{1}(\xi)+\left\|\lambda^{*}\right\| E C_{2}(\xi)+3 E R(\xi)+\gamma_{3}+\left\|\lambda^{*}\right\| \gamma_{4}+3 \gamma_{5},
\end{aligned}
$$

положительно. Тогда существует множество $\mathfrak{B}\left(\gamma_{1}, \ldots, \gamma_{6}\right) \subset R^{s}$ такое, чTO

1) если $\xi \in \mathfrak{B}\left(\gamma_{1}, \ldots, \gamma_{6}\right)$, то задача (1) имеет решение $x^{*}(\xi)$,

2) вероятность

$$
P\left[\xi \in \mathfrak{B}\left(\gamma_{1}, \ldots, \gamma_{6}\right)\right] \geqslant q\left(\gamma_{1}, \ldots, \gamma_{6}\right),
$$

3) вероятность

$$
\begin{gathered}
P\left[\xi \in\left\{\xi:\left\|x^{*}(\xi)-x^{*}\right\|<\varepsilon\right\} \cap \mathfrak{B}\left(\gamma_{1}, \ldots, \gamma_{6}\right)\right] \geqslant \\
\geqslant 1-\left[\frac{D_{1}}{\gamma_{1}^{2}}+\frac{D_{2}}{\gamma_{2}^{2}}+\frac{\psi_{1}}{\gamma_{3}^{2}}+\frac{\psi_{2}}{\gamma_{4}^{2}}+\frac{\psi_{3}}{\gamma_{5}^{2}}+\right. \\
\left.+\frac{\sum_{i=m_{1}+1}^{m} \sigma^{2}\left[g_{i}\left(x^{*}, \xi\right)\right]}{\left(1-\gamma_{6}\right)^{2}\left|\max _{i=m_{1}+1, \ldots, m} E g_{i}\left(x^{*}, \xi\right)\right|^{2}}\right]-\frac{c_{1}^{2}}{\varepsilon^{2}}\left(\sqrt[3]{D_{3}}+\sqrt[3]{\left\|\lambda^{*}\right\|^{2} D_{2}}+\sqrt[3]{D_{4}}\right)^{3}
\end{gathered}
$$

для любого $\varepsilon$,

$$
\begin{gathered}
0<\varepsilon \leqslant \min \left[\gamma_{6}\left|E g_{i}\left(x^{*}, \xi\right)\right| / K, \quad i=m_{1}+1, \ldots, m, \lambda_{j}^{*},\right. \\
\left.j=1, \ldots, m_{1}, 1 /\left(4 c_{1} c_{2}\right)\right] .
\end{gathered}
$$

Доказательство. Рассмотрим систему

$$
\begin{aligned}
& g_{i}(x, \xi)=0, \\
& f^{\prime}{ }_{x}(x, \xi)+\sum_{i=1}^{m_{1}} \lambda_{i} g_{i x}(x, \xi)=0
\end{aligned}
$$

относительно $x=\left(x_{1} \ldots x_{n}\right)^{\mathrm{T}}$ и $\lambda=\left(\lambda_{1} \ldots \lambda_{m_{1}}\right)^{\mathrm{T}}$. В теореме $3\left[{ }^{1}\right]$ приведены условия, при выполнении которых система (7) имеет в случае предположений $1-5$ решение $x^{*}(\xi)=\left(x_{1}{ }^{*}(\xi) \ldots x_{n}{ }^{*}(\xi)\right)^{\mathrm{T}}, \quad \lambda^{*}(\xi)=$ $=\left(\lambda_{1}{ }^{*}(\xi) \ldots \lambda_{m_{1}}{ }^{*}(\xi)\right)^{\mathrm{T}}$ с положительной вероятностью, не меньшей числа

$$
\begin{gathered}
p\left(\gamma_{1}, \ldots, \gamma_{5}\right)=1-\left[\frac{D_{1}}{\gamma_{1}^{2}}+\frac{D_{2}}{\gamma_{2}^{2}}+\frac{\psi_{1}}{\gamma_{3}^{2}}+\frac{\psi_{2}}{\gamma_{4}^{2}}+\frac{\psi_{3}}{\gamma_{5}^{2}}+\right. \\
\left.+144 \tilde{c}_{1}^{4} c_{2}^{2}\left(D_{3}+\left\|\lambda^{*}\right\|^{2} D_{2}+D_{4}\right)\right] .
\end{gathered}
$$

Другими словами, в $\left[{ }^{1}\right]$ указано такое множество $\mathfrak{U}\left(\gamma_{1}, \ldots, \gamma_{5}\right) \subset R^{s}$, что при каждом $\xi \in \mathfrak{U}\left(\gamma_{1}, \ldots, \gamma_{5}\right)$ существует решение $x^{*}(\xi), \lambda^{*}(\xi)$ системы (7),

$$
P\left[\xi \in \mathfrak{U}\left(\gamma_{1}, \ldots, \gamma_{5}\right)\right] \geqslant p\left(\gamma_{1}, \ldots, \gamma_{5}\right)
$$

и

$$
\begin{gathered}
P\left[\xi \in\left\{\xi:\left\|x^{*}(\xi)-x^{*}\right\|<\varepsilon\right\} \cap \mathcal{U}\left(\gamma_{1}, \ldots, \gamma_{5}\right)\right] \geqslant \\
\geqslant p\left(\gamma_{1}, \ldots, \gamma_{5}\right)-\left(9 \widetilde{c}_{1}^{2}\left(D_{3}+\left\|\lambda^{*}\right\|^{2} D_{2}+D_{4}\right)\right) / \varepsilon^{2}
\end{gathered}
$$

для любого $\varepsilon>0$. Здесь $\tilde{c}_{1}=\alpha_{1}+\alpha_{1}^{2} \alpha_{2}^{2} \alpha_{3}+2 \alpha_{1} \alpha_{2} \alpha_{3}+\alpha_{3}$. В настоящей работе использованы вместо оценок (8), (9) улучщенные оценки 


$$
\begin{gathered}
P\left[\xi \in \mathfrak{U}\left(\gamma_{1}, \ldots, \gamma_{5}\right)\right] \geqslant 1-\left[\frac{D_{1}}{\gamma_{1}^{2}}+\frac{D_{2}}{\gamma_{2}^{2}}+\frac{\psi_{1}}{\gamma_{3}^{2}}+\frac{\psi_{2}}{\gamma_{4}^{2}}+\frac{\psi_{3}}{\gamma_{5}^{2}}+\right. \\
\left.+16 c_{1}^{4} c_{2}^{2}\left(\sqrt[3]{D_{3}}+\sqrt[3]{\left\|\lambda^{*}\right\|^{2} D_{2}}+\sqrt[3]{D_{4}}\right)^{3}\right]
\end{gathered}
$$

и

$$
\begin{gathered}
P\left[\xi \in\left\{\xi:\left\|x^{*}(\xi)-x^{*}, \lambda^{*}(\xi)-\lambda^{*}\right\|<\varepsilon\right\} \cap \mathcal{U}\left(\gamma_{1}, \ldots, \gamma_{5}\right)\right] \geqslant \\
\geqslant 1-\left[\frac{D_{1}}{\gamma_{1}^{2}}+\frac{D_{2}}{\gamma_{2}^{2}}+\frac{\psi_{1}}{\gamma_{3}^{2}}+\frac{\psi_{2}}{\gamma_{4}^{2}}+\frac{\psi_{3}}{\gamma_{5}^{2}}+\frac{c_{1}^{2}}{\varepsilon^{2}}\left(\sqrt[3]{D_{3}}+\sqrt[3]{\left\|\lambda^{*}\right\|^{2} D_{2}}+\sqrt[3]{D_{4}}\right)^{3}\right]
\end{gathered}
$$

для любого $\varepsilon, 0<\varepsilon \leqslant 1 /\left(4 c_{1} c_{2}\right)$, которые получены аналогичным [ $\left.{ }^{1}\right]$ способом (их вывод здесь опускаем). Кроме того, вместо входящей в (8) и (10) постоянной $\tilde{c}_{1}$ в (11) и (12) использована постоянная $c_{1}$, полученная из более точной оценки нормы блочной матрицы

$$
\left\|\left(\begin{array}{cc}
l_{x x}^{\prime \prime}\left(x^{*}, \lambda^{*}, \xi\right) & {\left[\begin{array}{c}
\left.g^{1 \ldots m_{1}^{\prime}}\left(x^{*}, \xi\right)\right]^{\mathrm{T}} \\
g_{x}^{1 \ldots m_{1}^{\prime}}\left(x^{*}, \xi\right)
\end{array}\right.}
\end{array}\right)\right\|=\left\|\left(\begin{array}{cc}
\mathcal{L}_{11}(\xi) & \mathcal{L}_{12}(\xi) \\
\mathcal{L}_{12}^{\mathrm{T}}(\xi) & \mathcal{L}_{22}(\xi)
\end{array}\right)\right\| \leqslant
$$

$\leqslant c_{1}=\max \left[\left\|\mathfrak{L}_{11}(\xi)\right\|,\left\|\mathfrak{L}_{22}(\xi)\right\|\right]+\left\|\mathfrak{L}_{12}(\xi)\right\| \leqslant$

$\leqslant\left\|\mathfrak{L}_{11}(\xi)\right\|+2\left\|\mathfrak{L}_{12}(\xi)\right\|+\left\|\mathfrak{L}_{22}(\xi)\right\|=\tilde{c}_{1}, \quad l(x, \lambda, \xi)=f(x, \xi)+\sum_{i=1}^{m_{1}} \lambda_{i} g_{i}(x, \xi)$.

Итак, с вероятностью, не меньшей $P\left[\xi \in \mathfrak{U}\left(\gamma_{1}, \ldots, \gamma_{5}\right)\right]$, система (7) имеет решение $x^{*}(\xi), \lambda^{*}(\xi)$ :

$$
\begin{gathered}
g_{i}\left(x^{*}(\xi), \xi\right)=0, \\
f_{x}^{\prime}\left(x^{*}(\xi), \xi\right)+\sum_{i=1}^{m_{1}} \lambda_{i}^{*}(\xi) g_{i x}^{\prime}\left(x^{*}(\xi), \xi\right)=0 .
\end{gathered}
$$

В предположении, что $g^{1 \ldots m_{1}}\left(x^{*}(\xi), \xi\right)$ имеет полный ранг, условия (13) необходимы для того, чтобы $x^{*}(\xi)$ было решением задачи

$$
\min _{x}\left\{f(x, \xi) \mid g^{1 \ldots m_{1}}(x, \xi)=0\right\} .
$$

В доказательстве теоремы $3\left[{ }^{1}\right]$ показано, что само строение множества $\mathfrak{U}\left(\gamma_{1}, \ldots, \gamma_{5}\right)$ обеспечивает матрице $g^{1 \ldots m_{1}}\left(x^{*}(\xi), \xi\right)$ действительно полный ранг. Кроме того, в точке $x^{*}(\xi)$ выполняются достаточные условия для решения задачи (14) и $x^{*}(\xi), \lambda^{*}(\xi)$ является седловой точкой функции $l(x, \lambda, \xi)=f(x, \xi)+\sum_{i=1}^{m_{1}} \lambda_{i} g_{i}(x, \xi)$. Легко видеть $\left[{ }^{3}\right]$, что если $\lambda_{i}{ }^{*}(\xi) \geqslant 0, i=1, \ldots, m_{1}$, то $x^{*}(\xi), \lambda^{*}(\xi)$ - седловая точка функции Лагранжа задачи

$$
\min _{x}\left\{f(x, \xi) \mid g^{1 \ldots m_{1}}(x, \xi) \leqslant 0\right\}
$$

и, следовательно, $x^{*}(\xi)$ есть ее решение. Таким образом, $x^{*}(\xi)$ является решением задачи (1), если
1) $\lambda_{i}^{*}(\xi) \geqslant 0, \quad i=1, \ldots, m_{1}$,
2) $g_{i}\left(x^{*}(\xi), \xi\right) \leqslant 0, \quad i=m_{1}+1, \ldots, m$.

Для того чтобы все $\lambda_{i}{ }^{*}(\xi), i=1, \ldots, m_{1}$, были неотрицательными, до- 
статочно, чтобы $\left\|\lambda^{*}-\lambda^{*}(\xi)\right\| \leqslant \min _{i=1, \ldots, m_{1}} \lambda_{i}^{*}$. Предположением 1 гарантировано, что $\min _{i=1, \ldots, m_{1}} \lambda_{i}^{*}>0$. Аналогично, для того чтобы $g_{i}\left(x^{*}(\xi), \xi\right) \leqslant 0$, $i=m_{1}+1, \ldots, m$, достаточно, чтобы

$$
\left\|g^{m_{1}+1 \ldots m}\left(x^{*}(\xi), \xi\right)-\left.E g^{m_{1}+1 \ldots}\right|^{m}\left(x^{*}, \xi\right)\right\| \leqslant\left|\max _{i=m_{1}+1, \ldots, m} E g_{i}\left(x^{*}, \xi\right)\right| .
$$

Поскольку

$$
\left\|g^{m_{1}+1 \ldots m}\left(x^{*}(\xi), \xi\right)-E g^{m_{1}+1 \ldots m}\left(x^{*}, \xi\right)\right\| \leqslant
$$

$\leqslant\left\|g^{m_{1}+1 \ldots m}\left(x^{*}(\xi), \xi\right)-g^{m_{1}+1 \ldots m}\left(x^{*}, \xi\right)\right\|+\| g^{m_{1}+1 \ldots m}\left(x^{*}, \xi\right)-$

$-E g^{m_{1}+1 \ldots m}\left(x^{*}, \xi\right)\|\leqslant K\| x^{*}(\xi)-x^{*}\|+\| g^{m_{4}+1 \ldots m}\left(x^{*}, \xi\right)-E g^{m_{1}+1 \ldots m}\left(x^{*}, \xi\right) \|$, то (15) выполняется, если $\left\|x^{*}(\xi)-x^{*}\right\| \leqslant \gamma_{6}\left|\max _{i=m_{1}+1, \ldots, m} E g_{i}\left(x^{*}, \xi\right)\right| / K$

и $\quad\left\|g^{m_{1}+1 \ldots m}\left(x^{*}, \xi\right)-E g^{m_{1}+1 \ldots m}\left(x^{*}, \xi\right)\right\| \leqslant\left(1-\gamma_{6}\right) \underset{i=m_{1}+1, \ldots, m}{\max _{1}} E g_{i}\left(x^{*}, \xi\right) \mid$

для некоторого $\gamma_{6}, 0<\gamma_{6}^{\prime}<1$. Следовательно, для того чтобы задача (1) имела решение, достаточно, чтобы одновременно:

1) $\xi \in \mathfrak{U}\left(\gamma_{1}, \ldots, \gamma_{5}\right)$; это обеспечивает существование решения $x^{*}(\xi)$, $\lambda^{*}(\xi)$ системы (7),

2) $\left\|\lambda^{*}-\lambda^{*}(\xi)\right\| \leqslant \min _{i=1, \ldots, m_{1}} \lambda_{i}^{*}$,

3) $\left\|x^{*}(\xi)-x^{*}\right\| \leqslant \gamma_{6}\left|\max _{i=m_{1}+1, \ldots, m} E g_{i}\left(x^{*}, \xi\right)\right| / K$,

4) $\left\|g^{m_{1}+1 \ldots m}\left(x^{*}, \xi\right)-E g^{m_{1}+1 \ldots m}\left(x^{*}, \xi\right)\right\| \leqslant\left(1-\gamma_{6}\right)\left|\max _{i=m_{1}+1 \ldots m} E g_{i}\left(x^{*}, \xi\right)\right|$.

Поскольку в силу (12)

$P\left[\xi \in \mathcal{H}_{1}\left(\gamma_{6}\right)\right]=P\left[\xi \in\left\{\xi:\left\|x^{*}(\xi)-x^{*}, \lambda^{*}(\xi)-\lambda^{*}\right\| \leqslant\right.\right.$ $\left.\leqslant \min \left[\gamma_{6}\left|\max _{i=m_{1}+1, \ldots, m} E g_{i}\left(x^{*}, \xi\right)\right| / K, \min _{i=1, \ldots, m_{1}} \lambda_{i}^{*}, 1 /\left(4 c_{1} c_{2}\right)\right]\right\} \cap$ $\left.\cap \mathfrak{U}\left(\gamma_{1}, \ldots, \gamma_{5}\right)\right] \geqslant 1-\left[\frac{D_{1}}{\gamma_{1}^{2}}+\frac{D_{2}}{\gamma_{2}^{2}}+\frac{\psi_{1}}{\gamma_{3}^{2}}+\frac{\psi_{2}}{\gamma_{4}^{2}}+\frac{\psi_{3}}{\gamma_{5}^{2}}+\right.$

$$
+\frac{c_{1}^{2}\left(\sqrt[3]{D_{3}}+\sqrt[3]{\left\|\lambda^{*}\right\|^{2} D_{2}}+\sqrt[3]{D_{4}}\right)^{3}}{\left\{\min \left[\gamma_{6}\left|\max _{i=m_{1}+1, \ldots, m} E g_{i}\left(x^{*}, \xi\right)\right| / K, \min _{i=1, \ldots, m_{1}} \lambda_{i}^{*}, 1 /\left(4 c_{1} c_{2}\right)\right]\right\}^{2}},
$$

а в силу предположения 5 и леммы 1 [ $\left.{ }^{1}\right]$

$$
\begin{aligned}
P\left[\mathfrak{U}_{2}\left(\gamma_{6}\right)\right] & =P\left[\xi \in \left\{\xi:\left\|g^{m_{1}+1 \ldots m}\left(x^{*}, \xi\right)-E g^{m_{1}+1 \ldots m}\left(x^{*}, \xi\right)\right\| \leqslant\right.\right. \\
& \left.\left.\leqslant\left(1-\gamma_{6}\right)\left|\max _{i=m_{1}+1, \ldots, m} E g_{i}\left(x^{*}, \xi\right)\right|\right\}\right] \geqslant \\
& \geqslant 1-\frac{\sum_{i=m_{1}+1}^{m} \sigma^{2}\left[g_{i}\left(x^{*}, \xi\right)\right]}{\left(1-\gamma_{6}\right)^{2}\left[\max _{i=m_{1}+1, \ldots, m} E g_{i}\left(x^{*}, \xi\right)\right]^{2}}
\end{aligned}
$$

то по лемме $4\left[{ }^{1}\right]$ для вероятности того, что ह принадлежит множеству $\mathfrak{B}\left(\gamma_{1}, \ldots, \gamma_{6}\right)=\mathfrak{u}\left(\gamma_{1}, \ldots, \gamma_{5}\right) \cap \mathfrak{U}_{1}\left(\gamma_{6}\right) \cap \mathfrak{U}_{2}\left(\gamma_{6}\right)$, получим оценку (4)(5). Оценка (6) непосредственно следует из (12). 


\section{Л И ТЕР А Т У Р А}

1. T a m m, E., Math. Operationsforsch. Statist., Ser. Optimization, 11, № 3, 487497 (1980).

2. Иоффе А. Д., Тихоми ро в В. М., Теория экстремальных задач, М., «Наука», 1974.

3. З ангв и лл У. И., Нелинейное программирование, М., «Сов. радио», 1973.

Институт кибернетики

Академии наук Эстонской ССР
Поступила в редакцию 16/III 1981

Ebu TAMM

\section{JUHUSLIKUST PARAMEETRIST SOLTUVA MITTELINEAARSE PLANEERIMISULESANDE LAHENDUVUSEST}

Käsitletavat ülesannet on vaadeldud koos niisuguse determineeritud ülesandega, mille sihifunktsioon ja kitsendused on esimese ülesande sihifunktsiooni ja kitsenduste matemaatilised ootused ja millel eeldatakse olevat lahend. On näidatud tingimused, millal juhuslikust parameetrist sōltuv ülesanne on lahenduv positiivse tōenäosusega, ning leitud selle tõenäosuse alumine tōke ja vaadeldud ülesannete lahendite vahelise kauguse tõenäosuslik hinnang.

\section{Ebu TAMM}

\section{ON SOLVABILITY OF THE NONLINEAR PROGRAMMING PROBLEM WITH A RANDOM PARAMETER}

In this article the nonlinear programming problem depending on a random vectorparameter $\xi$ is considered. It is treated in connection with another, deterministic problem, in which the goal function and restrictions are mathematical expectations of those in the first one. Existence of a solution $x^{*}$ of this deterministic problem is assumed. The conditions laid on the goal and restrictions of both problems require mainly some differentiability properties of these functions. The distribution of $\xi$ is restricted only by the existence of some second-order moments of some functions of $\xi$. A theorem that establishes the conditions under which the first problem has a solution $x^{*}(\xi)$ with positive probability, is proved. Besides, for the distance between $x^{*}$ and $x^{*}(\xi)$ a probabilistic estimate is found. The article is essentially based on the author's earlier paper [ $\left.{ }^{1}\right]$. 